\title{
First results with SiPM tiles for TOF PET based on FBK RGB-HD technology
}

\author{
Alessandro Ferri ${ }^{*}$, Fabio Acerbi ${ }^{1}$, Peter Fischer ${ }^{2}$, Alberto Gola ${ }^{1}$, Giovanni Paternoster ${ }^{1}$, Claudio Piemonte ${ }^{2}$, \\ Michael Ritzert ${ }^{2}$, Torsten Solf ${ }^{3}$, Illaria Sacco ${ }^{2}$, Nicola Zorzi ${ }^{1}$ \\ From PSMR 2015: 4th Conference on PET/MR and SPECT/MR \\ La Biodola, Isola d'Elba, Italy. 17-21 May 2015
}

${ }^{1}$ Fondazione Bruno Kessler, Trento,
Italy
We present the first results of timing and energy resolution of two newly developed tiles based on FBK RGB-HD SiPMs. The first tile has dimensions of $32 \times 32 \mathrm{~mm}^{2}$ and is composed of $8 \times 8 \mathrm{SiPMs}$, with a regular pitch of $4 \mathrm{~mm}$ and a cell size of $25 \times 25 \mu \mathrm{m}^{2}$. Although manufactured with a standard bond wire technology, the tile achieves a fill factor at the tile level of $85 \%$. We produced two versions: one with a single-ended and the other with differential readout. We tested the first prototypes with single-ended readout with a scintillator array, perfectly matching the tile pitch and composed of $8 \times 8$ LYSO crystals with dimensions of $4 \times 4 \times 22 \mathrm{~mm}^{3}$. First, we tested the tile using a singlechannel setup, based on a fast, discrete amplifier, a digital oscilloscope and a PC, reading one SiPM at a time. At $20{ }^{\circ} \mathrm{C}$, we measured an energy resolution of $10.7 \%$ FWHM. For the timing measurements we compared two conditions: when only one SiPM was biased and read, and when all the 64 SiPMs were biased but only one was read. At $20{ }^{\circ} \mathrm{C}$, we measured a timing resolution of 200 ps FWHM in the first case, and 220 ps FWHM in the second case. Then, we tested the whole tile with a dedicated ASIC (PETA3), and measured the energy and timing resolution of two tiles in coincidence. The second tile is composed of 144 SiPMs, mounted on a water cooled, ceramic LTCC substrate. On the top side, it contains $12 \times 12$ SiPMs with a regular pitch of $2.5 \mathrm{~mm}$. Also in this case, the SiPM technology is the RGB-HD with a cell size of $25 \times 25 \mu^{2}$. On the bottom side, four readout ASICs of the latest generation (PETA5) are flip-chip mounted. First results will be presented.

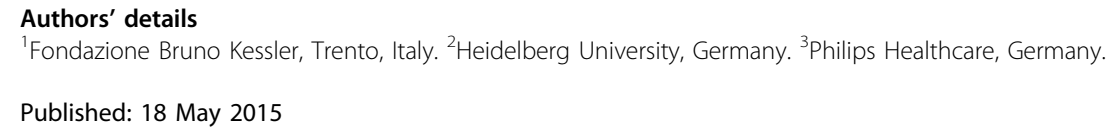

\section{SpringerOpen ${ }^{\circ}$}

(C) 2015 Ferri et al; licensee Springer. This is an Open Access article distributed under the terms of the Creative Commons Attribution License (http://creativecommons.org/licenses/by/4.0), which permits unrestricted use, distribution, and reproduction in any medium, provided the original work is properly cited. 